\title{
MORFOLOGÍA DEL APARATO REPRODUCTOR EN ODONTOTAENIUS STRIATOPUNCTATUS (PERCHERON, 1835) (COLEOPTERA: PASSALIDAE)
}

\author{
Magdalena Cruz Rosales ${ }^{1}$ y María Luisa CASTILlo² \\ 1 Biodiversidad y Ecología Animal, 2 Biología de Suelos, Instituto de Ecología, A.C. \\ Km 2.5 Carretera antigua a Coatepec, No. 351 Congregación El Haya, \\ Xalapa 91070, Ver. MÉXICO \\ magda.cruz@inecol.edu.mx, marilu.castillo@inecol.edu.mx
}

\begin{abstract}
RESUMEN
Se describe la anatomía e histología del aparato reproductor de hembras y machos de Odontotaenius striatopunctatus, especie de pasálido frecuente en bosques tropicales de Mesoamérica. El aparato reproductor de las hembras está constituido por un par de ovarios, cada uno con un par de ovariolas, dos oviductos laterales, un largo oviducto medio una cámara genital, una bursa copulatrix y una gran espermateca incluyendo su glándula. Los machos tienen un par de testículos, cada uno con un par de folículos testiculares, sus conductos deferentes, una vesícula seminal por cada lado, un conducto eyaculador y dos pares de glándulas accesorias. Se menciona la incidencia en los machos de variaciones en el número de folículos testiculares, y se pone en evidencia la presencia de espermatóforo en la familia Passalidae.

Palabras Clave: Odontotaenius striatopunctatus, Passalidae, aparato reproductor, anatomía, histología, espermatóforo.
\end{abstract}

\begin{abstract}
Female and male reproductive system anatomy of Odontotaenius striatopunctatus is described. This passalid species is common inside decomposition logs in the tropical forests of Mesoamerica. The female reproductive system consists of two ovaries with two ovarioles each, two lateral oviducts, a long oviduct, a genital chamber, a bursa copulatrix and a big spermatheca with its gland. The male reproductive system consists of two testes, each with a pair of testicular follicles and respective vas deferens, two seminal vesicles in each side, a long ductus eyaculatorius, the aedeagus, and two pairs of accessory glands. Two males were found with different numbers of testicular follicles. The spermatophore is described by first time for the family. Key Words: Odontotaenius striatopunctatus, Passalidae, reproductive system, anatomy, histology, spermatophore.
\end{abstract}

\section{INTRODUCCIÓN}

Los coleópteros de la familia Passalidae son insectos comunes en los troncos en descomposición de los ecosistemas tropicales húmedos, cuya importancia radica en 
ser organismos degradadores (Castillo \& Reyes-Castillo 2003). La estructura subsocial de los Passalidae se basa en el grupo familiar, formado por una pareja de adultos, macho y hembra, quienes brindan cuidados a la cría. Todo el ciclo de vida se desarrolla en el interior de los árboles muertos de donde salen en la etapa adulta para dispersarse (Reyes-Castillo \& Halffter 1983; Schuster \& Schuster 1997).

En relación a Odontotaenius striatopunctatus (Percheron), es una especie frecuente en los bosques tropicales de Mesoamérica, su rango altitudinal va desde el nivel del mar hasta cerca de $2100 \mathrm{~m}$, de tal manera que muestra una amplia variación en cuanto a preferencias de hábitat, tanto en bosques tropicales abiertos o perturbados y templados (Reyes-Castillo 1970), manteniendo como constante su presencia en lugares húmedos.

Pocos han sido los estudios que sobre diversos aspectos del aparato reproductor en Passalidae se han realizado. En cuanto a las hembras se ha reportado que tienen un par de ovarios, cada uno a su vez con un par de ovariolas (Robertson 1961, Reyes-Castillo \& Ritcher 1973, Ritcher \& Baker 1974). La genitalia interna de machos y hembras de tres especies africanas de Pentalobus fue descrita por Baker (1973). Virkki (1961) comparó la estructura del testículo de Passalus punctiger y Arrox agassizi con la de otros escarabajos, ubicando a los testículos de estas especies en un nivel primitivo, en relación a otras especies de la misma Superfamilia. En Odontotaenius disjunctus, se ha descrito la ovogénesis, (Bryan 1954) y la histología y el desarrollo gonádico de hembras y machos (Krause 1946, 1947). Fonseca y Buhrnheim (1984) realizaron un estudio en las ovariolas de las especies Passalus convexus y P. latifrons, para determinar su edad fisiológica. Recientemente Boucher (2005), en su amplio estudio sobre la evolución y filogenia de esta familia, realiza un interesante análisis de la genitalia de hembras y machos de diversas especies de Passalinae.

En este estudio presentamos una aportación al conocimiento de la morfología del aparato reproductor de hembras y machos de la especie del pasálido neotropical Odontotaenius striatopunctatus, destacando algunas observaciones particulares en las hembras, así como la incidencia de variaciones en el número de folículos testiculares de algunos machos. También se menciona por primera vez la presencia de espermatóforo en Passalidae.

\section{MATERIAL Y MÉTODOS}

Para este estudio se utilizaron 30 individuos, los cuales fueron obtenidos a partir de colectas hechas en octubre de 2001 en la localidad de Briones, y de la cría obtenida en el laboratorio con los individuos colectados. El lugar de colecta está ubicado a $3 \mathrm{~km}$ al SO de la ciudad de Xalapa, Estado de Veracruz, (19 $30^{\prime} 38^{\prime \prime} \mathrm{N}$, $96^{\circ} 57^{\prime} 03^{\prime}$ W), a $1300 \mathrm{~m}$ sobre el nivel del mar en un remanente de bosque mesófilo 
de montaña. Los individuos fueron colocados en terrarios de vidrio $(37 \times 28 \times 20 \mathrm{~cm})$ y mantenidos a $27^{\circ} \mathrm{C}, 80 \%$ de humedad relativa y 14 horas de luz, siguiendo el protocolo utilizado por Hernández-Martínez \& Castillo (2003).

Para la realización del estudio se contó con 17 hembras y 13 machos, los cuales fueron disecados en solución de Ringer para la obtención de las gónadas utilizando un estereomicroscopio SV-8 de Carl Zeiss ${ }^{\circledR}$. Las estructuras reproductoras se fijaron en solución de Carnoy por $24 \mathrm{hr}$ y después se mantuvieron en alcohol etílico de $96^{\circ}$. Se hicieron esquemas de las gónadas a escala y en fresco al momento de la disección. Algunas de estas gónadas de hembras y machos fueron teñidos in toto con la técnica de Feulgen-Verde luz; otras fueron procesadas para ser incluidas en parafinaceloidina, cortadas en serie a $8 \mu \mathrm{m}$ de grosor y teñidas con la técnica del Acido Peryódico Schiff (PAS)-Hematoxilina. Para definir algunas características de la intima cuticular del aparato reproductor de las hembras se realizó la técnica de aclaración y tinción con el Negro de Clorazol (Martínez 1999) en 5 de las 17 hembras estudiadas. En algunas hembras se separó y abrió por la mitad la espermateca para hacer un frotis de su contenido, el cual fue fijado con metanol, secado al aire y teñido con azul de toluidina. Las fotos se tomaron con un estereomicroscopio Nikon ${ }^{\circledR}$ Eclipse E-600, con cámara digital Nikon ${ }^{\circledR}$ Coolpix 990 de 3.34 Mega pixels.

\section{RESULTADOS}

Hembras. El aparato reproductor (Fig. 1A) está formado por dos ovarios, cada uno con un par de ovariolas. Las ovariolas de tipo telotrófico, al igual que en todos los coleópteros, están constituidas por su filamento terminal, el germario, el vitelario y un corto pedicelo (Fig. 2A). Dicho pedicelo desemboca al cálix, que continúa con el oviducto lateral (Fig. 2B). Los oviductos laterales son cortos y se unen para formar un largo oviducto medio hasta desembocar en la cámara genital (Fig. 2C). Junto a la cámara genital está la bursa copulatrix (Fig. 2D, E). La espermateca es grande con forma de un ovoide alargado (Fig. 2F), su glándula es tubular cuyo extremo ciego es más ancho que el resto de la glándula (Fig. 2G), el conducto de la espermateca desemboca en la cámara genital, entre la desembocadura de la bursa copulatrix y del oviducto común (Fig. 2D,E).

A nivel histológico cada ovariola está rodeada por una membrana o envoltura peritoneal, cuyo extremo apical forma el filamento terminal. Internamente entre el filamento y el germario se observa una zona con abundante material PAS + (Fig. 3B). En el germario, además de las numerosas ovogonias y células nutricias, se encuentran en la región basal pequeños ovocitos en crecimiento, dispersos entre abundantes células prefoliculares y pequeños gránulos PAS+ (Fig. 3A). En el vitelario de la mayoría de las hembras se observan uno o dos ovocitos por ovariola en pleno crecimiento (Fig. 2A). 


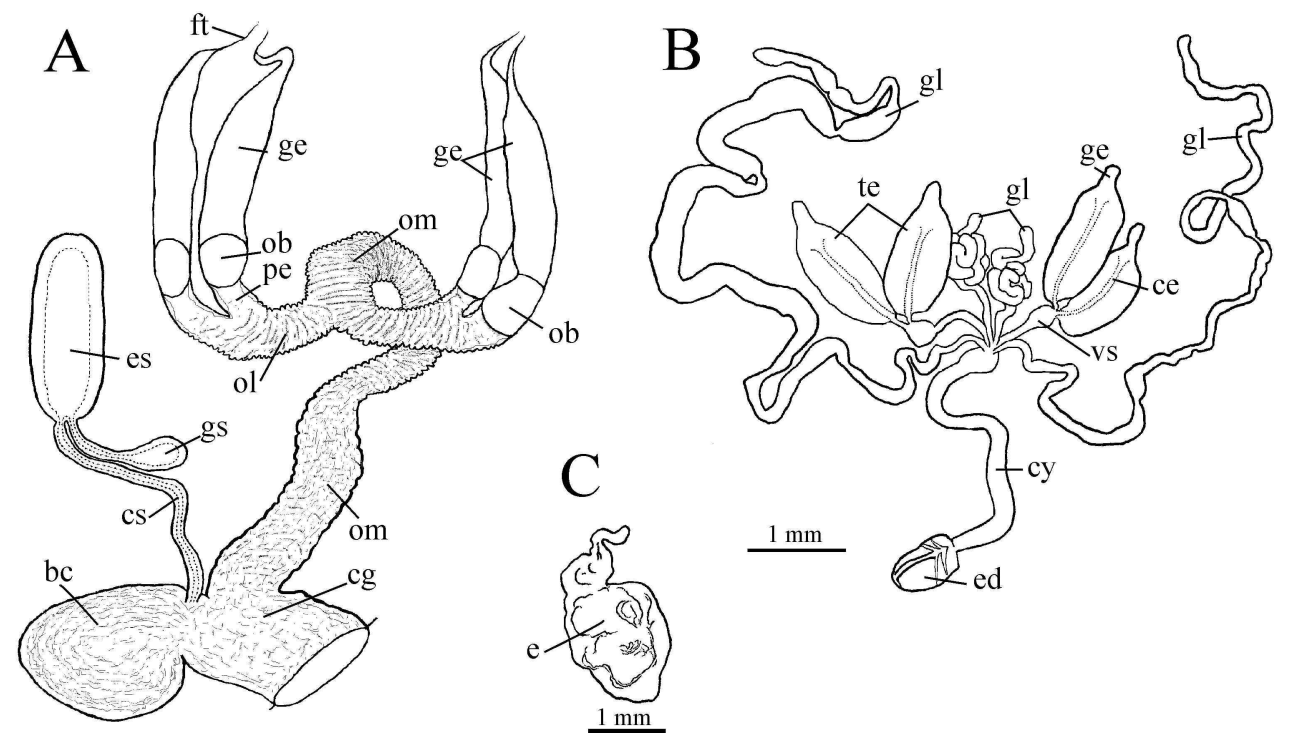

Figura 1. Esquemas del aparato reproductor de la hembra (A), del macho (B) y del espermatóforo (C) de Odontotaenius striatopunctatus. Abreviaciones utilizadas: bc- bursa copulatrix, ce- conducto eferente, cg- cámara genital, cs- conducto de la espermateca, cy- conducto eyaculador, ed- edeago, es- espermateca, ft- filamento terminal, ge- germario, gl- glándulas, gs- glándula de la espermateca, ob- ovocito basal, oc- oviducto común, ol- oviducto lateral, om- oviducto medio, pe- pedicelo, tefolículos testiculares, vs- vesícula seminal.

Cada ovocito está rodeado de células foliculares y entre el ovocito basal y el pedicelo se observa el tapón prefolicular muy evidente aún en material teñido in toto (Fig. 2A, 3A). El cálix es corto y bien definido por una gruesa capa epitelial y muscular. Este epitelio está formado de células altas, con núcleos compactos y de posición basal.

El oviducto lateral está formado externamente por una gruesa envoltura de células musculares principalmente de tipo circular. Su epitelio también es muy grueso y se pliega irregularmente, por lo que sus células son grandes e irregulares, con núcleos compactos y de posición basal (Fig. 3C). Los dos oviductos laterales se unen para formar un oviducto medio (Fig. 2B), que básicamente presenta la misma estructura histológica descrita para los oviductos laterales, pero con un mayor diámetro del conducto y grosor de su pared muscular (Fig. 2C). A la mitad de su trayecto desde la unión de los oviductos laterales y la desembocadura con la cámara genital, se observa un cambio en la estructura del oviducto. Aunque básicamente también está formado por una gruesa envoltura muscular, a partir de este nivel la íntima cuticular se hace presente. A este nivel el epitelio está constituido por células de cúbicas a romboidales, con núcleos grandes y 

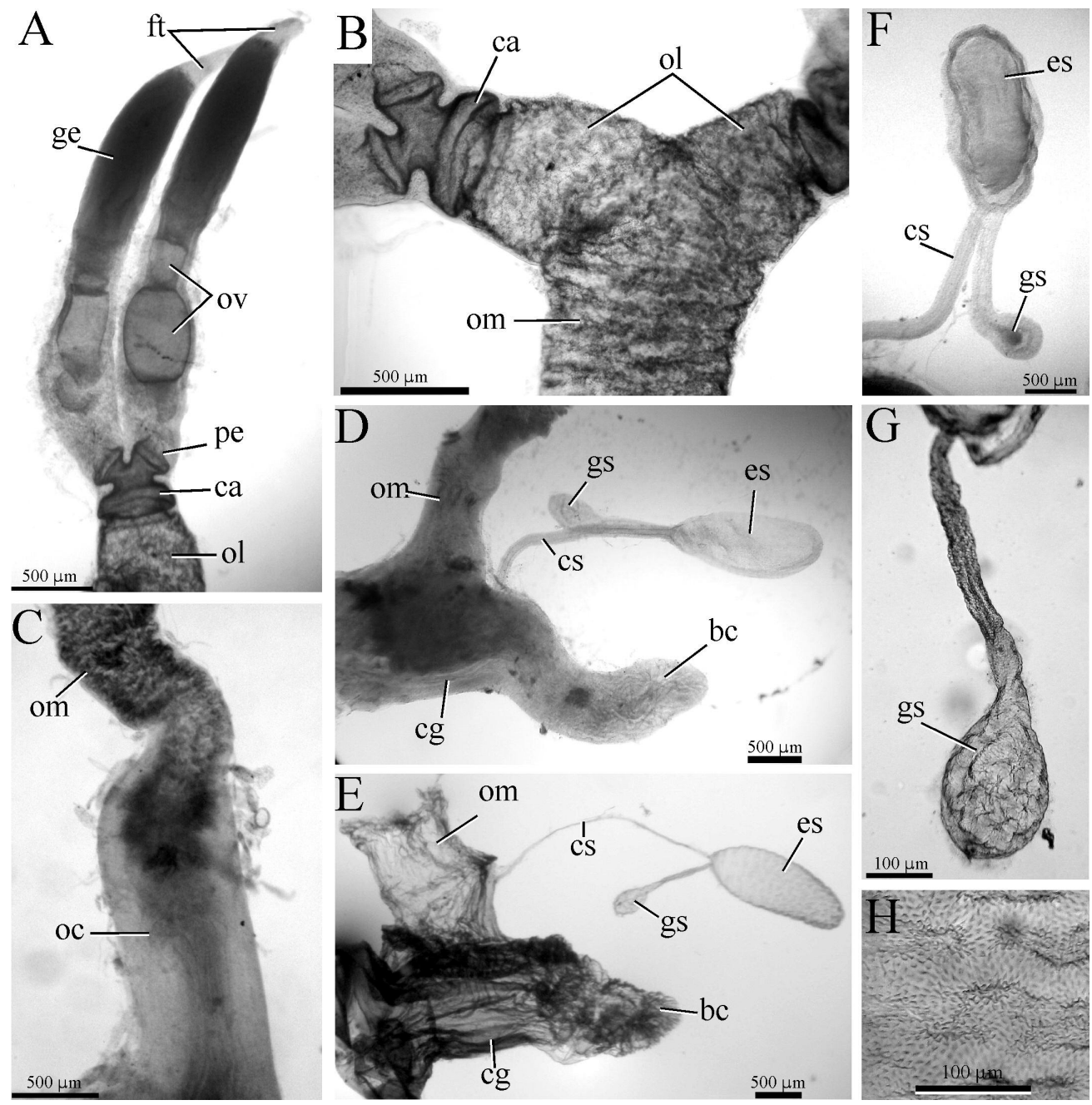

Figura 2. Microfotografías del aparato reproductor de hembras de O. striatopunctatus: (A) un ovario con su par de ovariolas, (B) unión de los oviductos laterales con el oviducto medio, (C) detalle de la zona media del oviducto, (D) cámara genital, bursa copulatrix y espermateca, (E) íntima cuticular de la cámara genital, oviducto medio, bursa copulatrix y espermateca, (F) espermateca, (G) íntima cuticular de la glándula de la espermateca, $(\mathrm{H})$ detalle de la íntima cuticular de la espermateca. Abreviaciones utilizadas: bc- bursa copulatrix, ca- calix, cg- cámara genital, cs- conducto de la espermateca, es- espermateca, ft- filamento terminal, ge- germario, gs- glándula de la espermateca, ol- oviducto lateral, om- oviducto medio, ov- ovocitos, pe- pedicelo. Técnica Feulgen-Verde Luz, excepto (E, G, H) con la Técnica del Negro de Clorazol. 
de posición central. Aparentemente la capa epitelial junto con su íntima cuticular son independientes de la capa muscular, la cual sirve para mantener reducido el diámetro del conducto, mientras que la capa epitelial está muy plegada a lo largo de su trayecto (Fig. 3D). Mediante la técnica del Negro del Clorazol se pudo observar fácilmente la íntima cuticular, resaltando así el gran diámetro del conducto cuando está distendido, además de ser evidente la textura fina y lisa de esta estructura (Fig. 2E).

La cámara genital o vagina está cubierta por una gruesa estructura muscular, cuya delgada capa epitelial sostiene una muy gruesa íntima cuticular (Fig. 2D,E, 3E). Contigua a la cámara genital se encuentra la bursa copulatrix. En 7 de las 17 hembras estudiadas se encontró un espermatóforo dentro de la bursa. La estructura de la bursa copulatrix es semejante a la de la cámara genital, pero con un mayor número de pliegues. El epitelio está formado de células cúbicas, con núcleos grandes de cromatina laxa y la íntima cuticular aunque es más delgada forma muchos más pliegues que en la cámara genital (Fig. 3E).

La espermateca (Fig. 2F,G) está constituida de una delgada envoltura muscular y un epitelio simple de células irregulares entre planas a cúbicas, cuya íntima cuticular es ligeramente rugosa, con una textura finamente espiniforme que se aprecia mejor después de aplicar la técnica del Negro de Clorazol (Fig. 2H). En el interior de la espermateca se observó abundante secreción PAS+ (Fig. 3F). La glándula de la espermateca es de forma tubular, en cuyo extremo ciego se encuentra principalmente su epitelio secretor, el cual está constituido de grandes células glandulares con forma irregular, que presentan núcleos grandes en posición central y abundantes secreciones citoplásmicas PAS+ (Fig. 3G). El conducto de la espermateca está constituido de una envoltura muscular gruesa y un epitelio formado de células compactas con núcleos pequeños en posición basal.

Machos. El aparato reproductor (Fig. 1B) está constituido por un par de testículos, cada uno con un par de folículos testiculares de forma ovoide alargada (Fig. 4A,B). De cada folículo parte su conducto deferente en cuyo trayecto se observa la vesícula seminal que tiene forma esférica. Posteriormente los conductos deferentes de cada lado desembocan en el conducto eyaculador, y éste a su vez desemboca en el edeago (Fig. 4C). Presenta dos pares de glándulas accesorias: un par más largo y con algunos abultamientos en su trayecto (Fig. 4D), cuyas secreciones al momento de la disección se observan casi transparentes, el segundo par de glándulas son más cortas y delgadas que se enrollan sobre sí mismas; sus secreciones se observan blanquecinas al momento de la disección. Los dos pares de glándulas también desembocan en el conducto eyaculador.

A nivel histológico los folículos testiculares presentan una organización radial bien definida por sus septos, con excepción del germario (Fig. 5A). Hacia la zona periférica del folículo se observan abundantes cistos de espermatocitos en maduración (Fig. 5B,C). En la zona central alrededor del conducto eferente, se observan cistos de espermátidas y espermatozoides en proceso de diferenciación y 

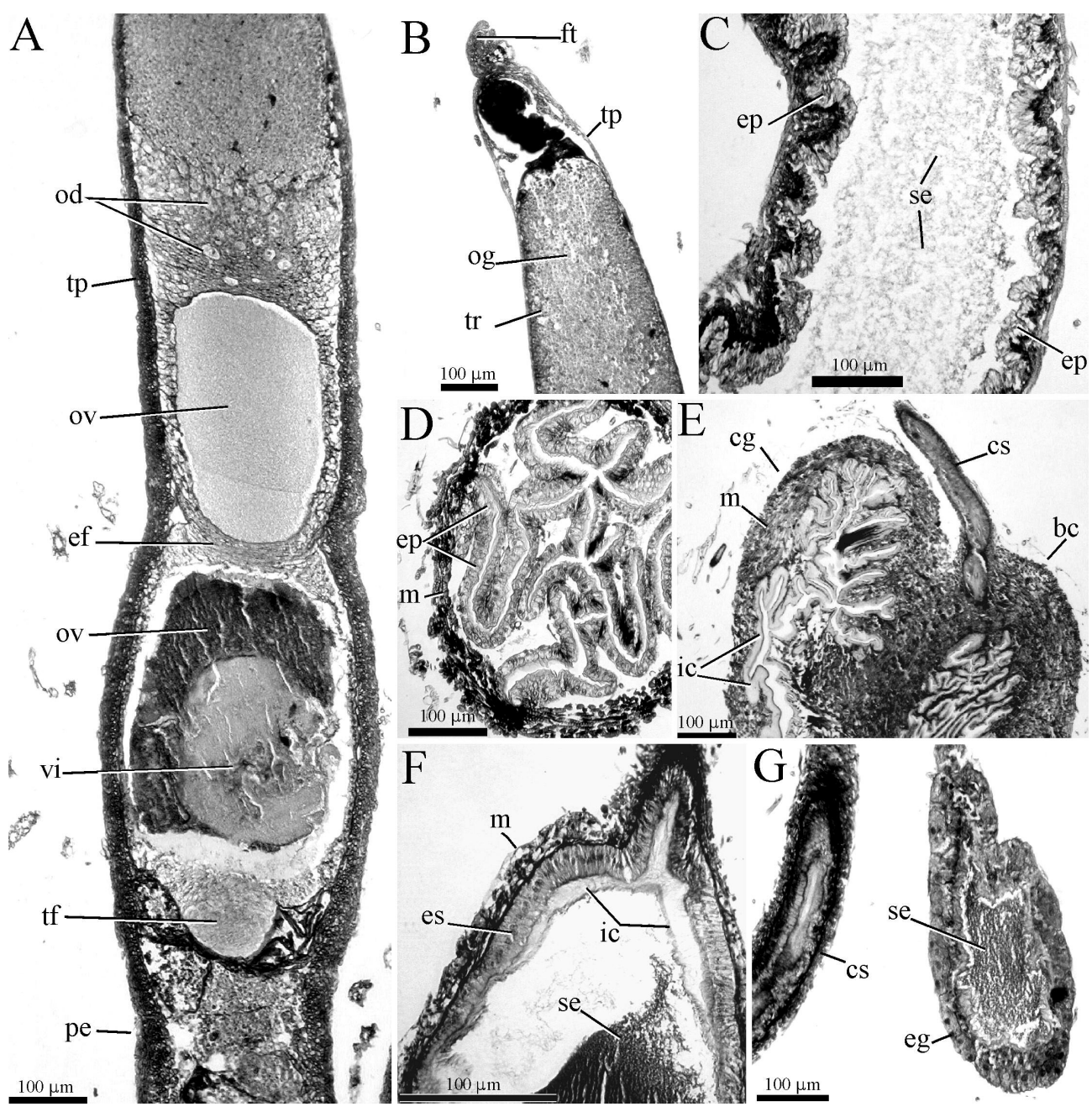

Figura 3. Microfotografías de cortes histológicos del aparato reproductor de hembras de $O$. striatopunctatus: (A) corte longitudinal de la zona media de una ovariola, (B) detalle de la zona apical del germario, (C) detalle del oviducto medio, (D) corte transversal del oviducto en su parte basal, (E) corte longitudinal de la cámara genital y bursa copulatrix, (F) corte longitudinal de la espermateca, (G) corte longitudinal de la glándula y conducto de la espermateca. Abreviaciones utilizadas: bcbursa copulatrix, cg- cámara genital, cs- conducto de la espermateca, ef- células foliculares, egcélulas glandulares de la espermateca, ep- epitelio del oviducto, es- epitelio de la espermateca, ftfilamento terminal, ic- íntima cuticular, m- células musculares, od- ovocitos en desarrollo, ogovogonias, ov- ovocito, pe- pedicelo, se- secreciones, tf- tapón folicular, tp- túnica propia, trtrofocitos, vi- vitelo. Técnica PAS-Hematoxilina. 

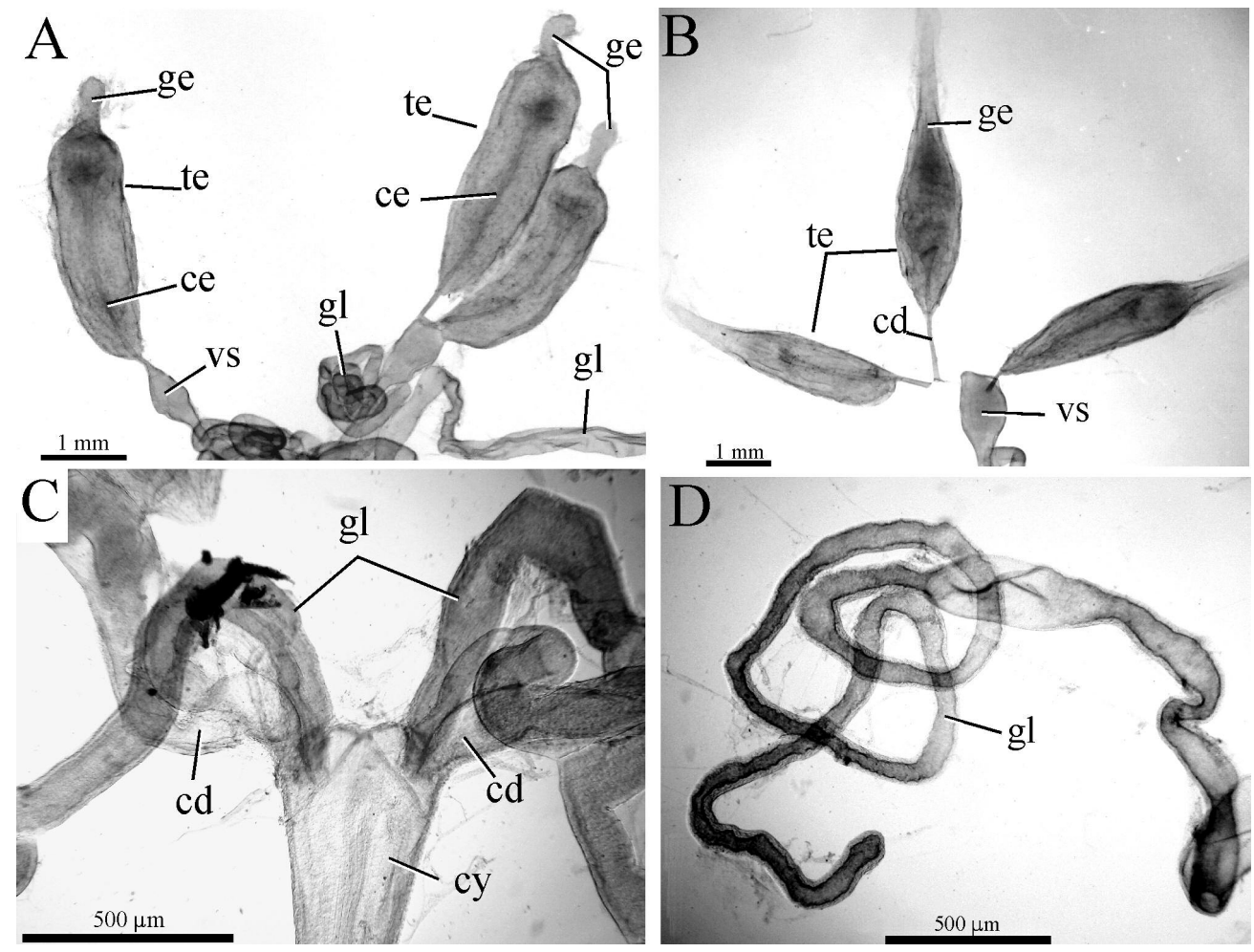

Figura 4. Microfotografías del aparato reproductor de machos de O. striatopunctatus: (A) folículos testiculares de machos con 1 y 2 folículos por testículo, (B) con 3 y 2 folículos por testículo (solo foto de los 3 folículos), (C) área de unión del conducto eyaculador con las glándulas accesorias y los conductos deferentes, (D) detalle de una de las glándulas accesorias. Abreviaturas utilizadas: cdconducto deferente, ce- conducto eferente, cy- conducto eyaculador, ge- germario, gl- glándulas accesorias, te- folículos testiculares, vs- vesícula seminal. Técnica Feulgen-Verde Luz.

maduración (Fig. 5C,D). Los espermatozoides en maduración se observan orientados paralelamente al conducto eferente y en sentido apical hacia la entrada del conducto eferente. En algunos machos se observó además una masa de células nutricias que forman un tapón sobre la entrada del conducto eferente. Por encima de esta entrada, en algunos casos se observó una masa abundante de tejido laxo y poco definido que aparentemente está en proceso de degeneración (Fig. 5B). Dentro del conducto eferente se observan algunas células nutricias dispersas entre los espermatozoides y abundante material PAS+ (Fig. 5D).

La vesícula seminal de forma esférica está cubierta por una envoltura muscular de varias células de grosor y un epitelio simple de células que varían de forma desde 

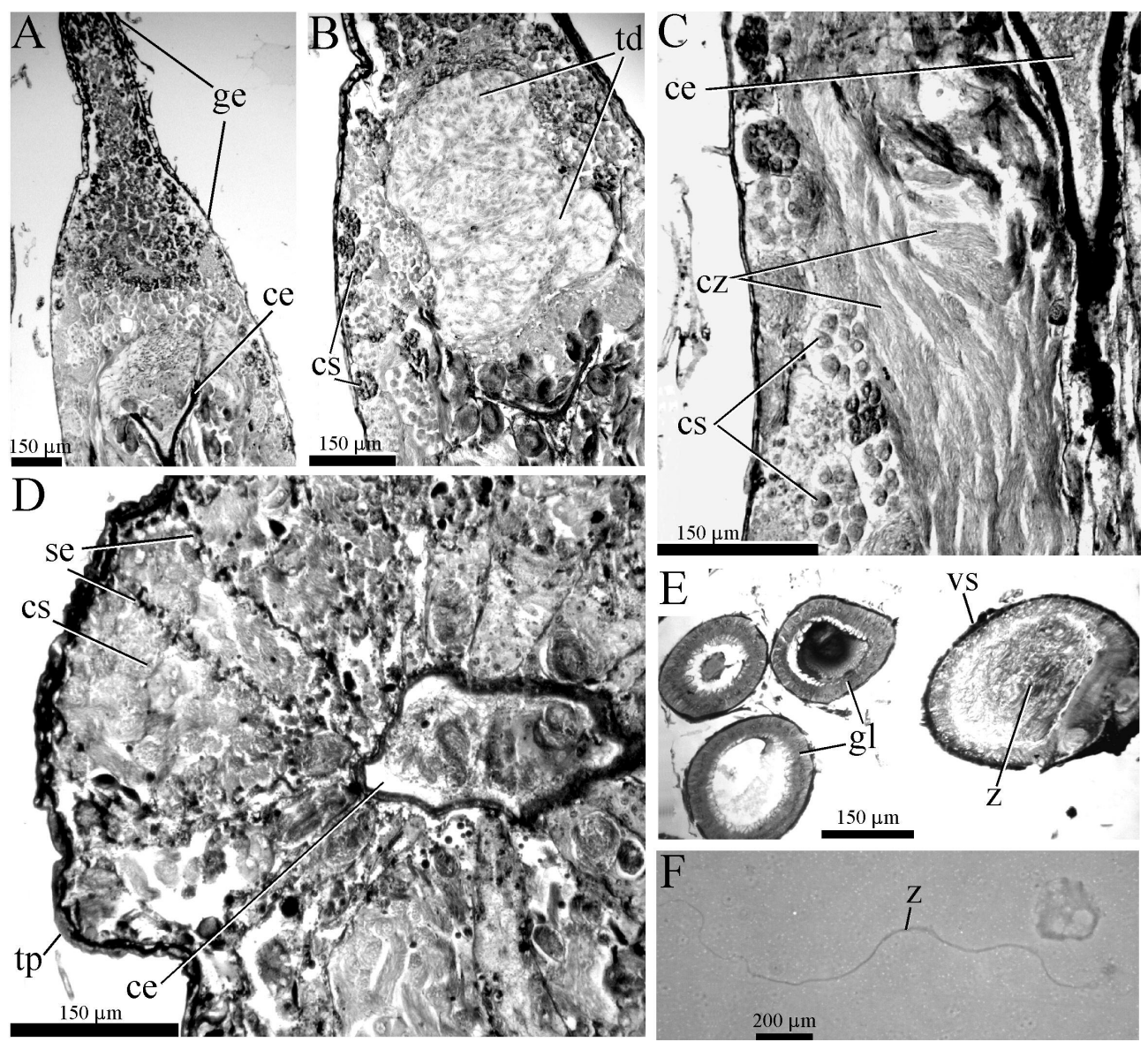

Figura 5. Microfotografías de cortes histológicos del aparato reproductor de machos de $O$. striatopunctatus: (A) corte longitudinal del germario y ápice del folículo testicular, (B) detalle de tejido en degeneración del folículo testicular, (C) detalle de diferentes cistos con espermatocitos y espermátidas, (D) corte transversal del folículo testicular a nivel de la entrada del conducto eferente, (E) corte transversal de las glándulas accesorias y de la vesícula seminal, (F) espermatozoide obtenido de frotis de la vesícula seminal. Abreviaturas utilizadas: ce- conducto eferente, cs- cistos con espermatocitos en diferente grados de madurez, cz- cistos cos espermatidas, ge- germario, gl- glándula accesoria, se- septos del folículo testicular, td- tejido en degeneración, tp- túnica propia, vs- vesícula seminal, z- espermatozoides. Técnica PAS-Hematoxilina, excepto F, técnica de Azul de Metileno en contraste de fases.

cúbicas a cilíndricas, sus núcleos son compactos y generalmente de posición basal, mientras que en el citoplasma se observan abundantes granulaciones apicales PAS+. Dentro de la vesícula se observan abundantes espermatozoides en desorden (Fig. 5E). 
Mediante el frotis del contenido de la vesícula seminal se pudieron observar largos espermatozoides, que en promedio miden $1.3 \mathrm{~mm}$ de longitud $(\mathrm{n}=8)$ (Fig. 5F).

Las glándulas accesorias se diferencian por su longitud, presencia o no de abultamientos en su trayecto, el grosor de sus paredes y por la cantidad y apariencia de sus secreciones. La pared epitelial simple de las glándulas está constituida de células planas con núcleos grandes de cromatina dispersa y citoplasma con granulaciones finas PAS + , todas las secreciones tienen una reacción PAS+, además de ser abundantes en el interior de sus conductos (Fig. 4D, 5E).

En 2 de los 13 machos estudiados se encontraron variaciones en el número de folículos testiculares. Cuando de manera normal se presenta un par de folículos testiculares por lado (2-2) en la familia Passalidae, en uno de los machos se encontraron 2-1 folículos testiculares y en otro macho 2-3 folículos testiculares por lado respectivamente. Estos folículos no presentaron diferencias anatómicas ni histológicas en comparación con lo observado en los folículos de los otros machos (Fig. 4A,B).

El espermatóforo (Fig. 1C) encontrado en 7 de las 17 hembras estudiadas, tiene una estructura compacta de textura rugosa, coloración blanquecina cuando está en fresco, con forma ovoide y un extremo más alargado. Su tamaño varió de 0.8 a 1.33 $\mathrm{mm}$ de ancho, por 1.12 a $1.87 \mathrm{~mm}$ de largo $(\mathrm{n}=6)$.

\section{DISCUSIÓN}

La anatomía del aparato reproductor de las hembras de Odontotaenius striatopunctatus, es semejante a la descrita para Odontotaenius disjunctus (Klause 1946, citado como Passalus cornutus), Pentalobus palini, P. barbatus y P. savagei (Baker 1973), Passalus convexus (Fonseca 1981), y Passalus latifrons (Fonseca \& Buhrnheim 1984). La composición de un par de ovariolas por ovario observado en $O$. striatopunctatus, ha sido considerada como un carácter constante en la familia Passalidae de acuerdo a Ritcher y Baker (1974), sin embargo se han encontrado variaciones en su número; Fonseca (1981) reporta la presencia en dos hembras de Passalus convexus con 2-3 y 2-4 ovariolas por lado respectivamente. En cuanto a la estructura histológica de las ovariolas de $O$. striatopunctatus de igual manera es similar a la descrita para $O$. disjunctus por Bryan en 1954 (citado como Popilius disjunctus).

La estructura del oviducto es muy variable entre los insectos debido a su origen embrionario (Snodgrass 1935, Matsuda 1976). En el caso de O. striatopunctatus su oviducto tiene origen tanto mesodérmico como ectodérmico, pues la presencia de íntima cuticular a partir de la región media y basal del oviducto medio pone en evidencia su origen ectodérmico. Matsuda (1976) considera una tendencia entre los insectos más evolucionados a presentar oviductos principalmente de origen ectodérmico, incluyendo los oviductos laterales. En el caso de O. striatopunctatus esta tendencia no se observa ya que hay una mayor proporción del oviducto con 
origen mesodermal y solo la parte basal del oviducto medio es de origen ectodermal.

Con un enfoque morfológico y taxonómico, Boucher (2005) define y denomina las variaciones de diversos lóbulos vaginales, llamando "lóbulo proximal dorsal de la vagina" a lo que nosotros consideramos como bursa copulatrix, confirmándolo por el hecho de haber encontrado en $O$. striatopunctatus un espermatóforo dentro de esta estructura, lo que demuestra el papel que cumple ésta durante la cópula, al servir como una verdadera bolsa copuladora, de acuerdo a la definición de Snodgrass (1935).

En lo que respecta a la forma de la espermateca, se encuentran diferencias entre las hembras de O. striatopunctatus y las hembras de los Passalinae africanos Pentalobus palini, P. barbatus y P. savagei, estudiados por Baker (1973), en estas especies la espermateca es muy reducida, además el autor no reporta la presencia de la glándula anexa a la espermateca. Recientemente Boucher (2005) hace notar la gran variabilidad en cuanto a la forma y tamaño que pueden presentar tanto la espermateca como su glándula anexa en la familia Passalidae.

La íntima cuticular de $O$. striatopunctatus es lisa, con excepción del reservorio de la espermateca que tiene una textura espiniforme. Aunque Boucher (2005) observó y comparó las genitalias de numerosas hembras de diferentes especies de Passalinae, incluyendo $O$. striatopunctatus, no observó esta característica en la espermateca, pero si concluyó que la íntima cuticular de la vagina es lisa, sin marcada diferenciación y sin espículas ni microvellosidades.

En el estudio realizado de la espermateca de diversas especies de Scarabaeoidea por López-Guerrero y Halffter (2000) fue incluida la de O. striatopunctatus. Estos autores consideran a la espermateca de $O$. striatopunctatus como una estructura primitiva por su forma de ampolla y por carecer de músculo constrictor, por lo que aparentemente es una contradicción en Passalidae, el tener un comportamiento subsocial evolucionado y una glándula espermática simple. Pero al mismo tiempo, López-Guerrero y Halffter (op.cit.) lo justifican, considerando que "a pesar de la compleja conducta de cortejo que se presenta en la familia, el hecho de que la pareja presente una prolongada monogamía, y que permanezca en el nido la mayor parte del tiempo, garantizando su permanencia, el proceso de precedencia espermática no tendría una ventaja selectiva real", con lo cual estamos de acuerdo. Sin embargo, esta situación aunque parece contradictoria, no significa que un comportamiento reproductor evolucione a la par que todas las estructuras asociadas al aparato reproductor.

La anatomía general del aparato reproductor de los machos de $O$. striatopunctatus es semejante a la de $O$. disjuntus (Krause 1946), Passalus punctiger y Arrox agassizi (Virkki 1961). Sin embargo, a diferencia de lo que observamos en $O$. striatopunctatus, los folículos testiculares de Arrox agassizi no presentan septos al interior (Virkki 1961). En tres especies de Pentalobus se observan más diferencias, hay un par de testículos, uno por lado y presentan vesículas seminales (Baker 1973). 
Es evidente la variación que puede existir entre especies de distintas tribus dentro de la misma familia, a nivel del número y forma de los testículos o de sus folículos testiculares, incluyendo la presencia o no de vesículas seminales.

Tomando en cuenta la clasificación propuestas por Virkki (1957), en relación a los folículos testiculares de los Scarabaeidae, se considera como caracteres más desarrollados la forma redondeada y la presencia de septos intrafoliculares. Sin embargo, en el caso de Passalidae esta caracterización no es clara, pues ni la forma de los folículos testiculares, ni la presencia de septos es constante. Su forma varía de ovoide alargada en O. striatopunctatus, O. disjunctus (Krause 1946), Passalus punctiger y Arrox agassizi (Virkki 1961) a redonda en las especies africanas Pentalobus palini, P. baratus y P. savagei (Baker 1973). Mientras que la ausencia de septos sólo se ha observado en Arrox agassizi (Virkki 1961). En este sentido aún es incierta la posición de los Passalidae por lo que amerita más estudios.

En otros Scarabaeoidea se han observado variaciones en la forma y la estructura histológica de los folículos testiculares que caracterizan tanto a familias, subfamilias o tribus, entre los grupos más estudiados están los Scarabaeidae, Aphodiidae y Geotrupidae (Virkki, 1957, Pluot-Sigwalt \& Martínez, 1998, Martínez \& Cruz, 1999, Martínez 2001, Martínez et al. 2001). Sin embargo, la escasez de estudios anatómicos e histológicos del aparato reproductor en Passalidae, impiden aún poder establecer la existencia de un algún tipo general de estructura básica.

Hasta ahora no se había observado una variación en el número de folículos testiculares en machos de Passalidae. En cambio las variaciones en el número de ovarios si se ha observado entre algunas hembras de diversas especies de Scarabaeidae (Heymons 1930, Pluot 1979, Martínez \& Cruz, 998, Martínez et al. 1999) Aphodiidae (Martínez et al. 2001) e inclusive en una especie de Passalidae (Fonseca, 1981).

Dentro del folículo testicular de $O$. striatopunctatus el abundante tejido laxo observado entre la parte basal del germario y la entrada del conducto eferente de algunos machos, tiene similitud con el tejido en proceso de degeneración observado en los folículos testiculares de varias especies de Scarabaeidae (Benítez \& Martínez 1985, López-Guerrero \& Halffter 1991, Martínez \& Cruz 1992, Cruz \& Huerta 1998).

Datos sobre la presencia de dos pares de glándulas accesorias (un par más largo y grueso y otro par más delgado y enrollado) ya han sido mencionados en los pocos estudios que sobre machos se han realizado en Passalidae (Krause 1946, Virkki 1961, Baker 1973).

Hasta ahora no se había mencionado la existencia de espermatóforos en Passalidae (P. Reyes-Castillo, com. pers.). El espermatóforo encontrado en las hembras de $O$. striatopunctatus tiene forma acorde con el espacio disponible dentro de la bursa copulatrix, por lo que podemos suponer que es ahí donde se elabora durante el proceso de cópula.

Aún quedan muchas interrogantes por resolver sobre los aspectos anatómicos y funcionales del aparato reproductor en Passalidae, por lo cual es recomendable realizar más estudios para ampliar el conocimiento en estas interesantes especies. 


\section{AGRADECIMIENTOS}

Deseamos agradecer a la Dra. Gabriela Heredia, del Laboratorio de Micología, Departamento de Biología de Suelos del Instituto de Ecología A.C. por facilitarnos el equipo para la obtención de las fotografías. Agradecemos los útiles comentarios hechos al manuscrito por P. Reyes-Castillo, así como a las constructivas observaciones de los revisores y del Editor que nos ayudaron sustancialmente a mejorar este documento. Este trabajo se realizó en los Departamentos de Biodiversidad y Ecología Animal, y Biología de Suelos del Instituto de Ecología A.C. (20007-10126 y 20009-10020).

\section{LITERATURA CITADA}

Baker, W.V. 1973. The genitalia of three species of Pentalobus (Col: Passalidae). J. Nat. Hist., 7: 435-440.

Benitez, F. J. C. \& I. Martínez M. 1985. Evolución histológica testicular durante el ciclo reproductor de Onthophagus gazella Fabricius y Canthon cyanellus cyanellus LeConte (Coleoptera: Scarabaeidae). Folia Entomol. Mex., 64: 33-40.

Boucher, S. 2005. Évolution et phylogénie des Coléoptères Passalidae (Scarabaeoidea). Ann. Soc. Entomol. Fr. (n.s.) 41(3-4): 239-604.

Bryan, J. H. D. 1954. Cytological and cytochemical studies of oogenesis of Popilius disjunctus (Illiger) (Coleoptera -Polyphaga). Biol. Bull., 107: 64-79.

Castillo, M. L. \& P. Reyes-Castillo. 2003. Los Passalidae: coleópteros tropicales degradadores de troncos de árboles muertos. Pp. 233-258. In: Ecología del suelo de la selva Tropical Húmeda de México. J. Álvarez-Sánchez y E. Naranjo-García (Ed.). Instituto de Ecología, Instituto de Biología, UNAM, México.

Cruz R. M. \& C. Huerta C. 1998. Comportamiento y actividad reproductora de los machos de Copris insertus Say (Coleoptera Scarabaeidae: Scarabaeinae). Acta Zool. Mex. (n.s.) 74: 163-173.

Fonseca, C.R.V. 1981. Ovários anômalos em Passalus convexus Dalman, 1817 (Coleoptera: Passalidae). Acta Amazon., 11(4): 839-841.

Fonseca, C.R.V. \& P. F. Buhrnheim. 1984. Estimate of physiological age in females of Passalus (Insecta, Coleoptera). Acta Amazon., 14(1-2): 314-321.

Hernández-Martínez, G. \& M.L. Castillo. 2003. Modified logs as a means for rearing and observing sapwood/heartwood dwelling passalids (Coleoptera: Passalidae) in the laboratory. Coleopt. Bull., 57(2): 243-244.

Heymons, R. 1930. Über die Morphologie des Weiblichen Geschlechtsapparatus der Gattung Scarabaeus L. Z. Morphol. Ökol. Tiere, 18: 536-574.

Krause, J. B. 1946. The structure of the gonads of the wood-eating beetle, Passalus cornutus Fabricius. Ann. Ent. Soc. Amer., 39: 193-206.

- 1947. The development of the gonads of the wood-eating beetle, Passalus cornutus Fabricius. Ann. Ent. Soc. Amer., 40: 172-202.

López-Guerrero, Y. \& V. M. Halffter. 1991. Degeneración testicular en Cephalodesmius armiger Westwood durante la época de cuidados del nido (Coleoptera: Scarabaeidae: Scarabaeinae). Elytron 5: 63-70. 
López-Guerrero, Y. \& G. Halffter. 2000. Evolution of the spermatheca in Scarabaeoidea. Fragm. entomol. 32(2): 225-285.

Martínez, M. I. 1999. Estudio de la anatomía microscópica en insectos: Técnicas básicas. Folia Entomol. Mex., 105:65-76.

. 2001. La biología reproductiva en Aphodiidae: síntesis de datos conocidos (Coleoptera). Boll. Soc. entomol. Ital., 133(2):113-130.

. 2003. Spermatophore in Aphodiinae (Coleoptera: Scarabaeidae). Proc. Entomol. Soc. Wash., 105(4):982-989.

Martínez M. I. \& M. Cruz R. 1988. Comportamiento, glándulas accesorias y centros neuroendocrinos en machos de dos especies de Canthon (Coleoptera: Scarabaeinae). Acta Zool. Mex. (n.s.) 27: 1-19.

— 1992. L'Activité de l'appareil reproducteur mâle pendant la vie imaginale chez deux espèces de Canthon (Coleoptera: Scarabaeidae). Acta Zool. Mex. (n.s.) 49: 1-22.

. 1998. The abnormal presence of two ovaries in a Canthon cyanellus cyanellus LeConte female (Coleoptera: Scarabaeidae: Scarabaeinae). Proc. Entomol. Soc. Wash., 100(2): 269-274.

. 1999. Comparative morphological analysis of testis follicles in dung beetles (Coleoptera: Scarabaeidae: Scarabaeinae, Aphodiinae, Geotrupinae). Proc. Entomol. Soc. Wash., 191(4):804-815.

Martínez, M. I., C. Deloya \& M. Dellacasa. 2001. Anatomical and functional data on female and male reproductive systems of some dung beetle species of Aphodiinae and Eupariinae of Mexico (Coleoptera: Scarabaeoidea: Aphodiidae). Proc. Entomol. Soc. Wash., 103(1):227-248.

Martínez, M. I., P. González V. \& E. Morelli. 1999. Anormalidades en el aparato reproductor de una hembra de Onthophagus hirculus Mannh. (Coleoptera Scarabaeidae: Scarabaeinae). Acta Zool. Mex. (n.s.) 76:127-129.

Matsuda, R. 1976. Morphology and evolution of the insect abdomen. Pergamon Press, Oxford. 543 pp.

Pluot, D. 1979. Évolution régresive des ovarioles chez les coléoptères Scarabaeinae. Ann. Soc. Entomol. Fr., (N.S.) 15(3): 575-588.

Pluot-Sigwalt, D. \& I. Martínez M. 1998. Anatomie morpho-fonctionnelle de l'appareil génital mâle des coléopèteres Scarabaeoidea coprophages: donnés comparatives. Ann. Soc. Entomol. Fr., (N.S.) 34(4):419-444.

Reyes-Castillo, P. 1970. Coleoptera, Passalidae: morfología y división en grandes grupos géneros americanos. Folia Entomol. Mex. 20-22: 1-240.

Reyes-Castillo, P. \& G. Halffter. 1983. La structure sociale chez les Passalides. Bull. Soc. Entomol. Fr., 88:619-635.

Reyes-Castillo, P. \& P. O. Ritcher. 1973. Ovariole number in Passalidae (Coleoptera). Proc. Entomol. Soc. Wash., 75(4): 478-479.

Ritcher, P. O. \& C. W. Baker. 1974. Ovariole numbers in Scarabaeoidea (Coleoptera: Lucanidae, Passalidae, Scarabaeidae). Proc. Entomol. Soc. Wash., 76(4): 480-494.

Robertson, J.G. 1961. Ovariole numbers in Coleoptera. Can. J. Zool., 39: 245-263. 
Schuster, J. C. 1975. A comparative study of copulation in Passalidae (Coleoptera): new positions for beetles. Coleopt. Bull., 29(2): 75-81

Schuster, J.C. \& L.B. Schuster.1997. The Evolution of social behavior in Passalidae (Coleoptera). Pp. 260-269. In: J.C. Choe \& B.J. Crespi. (Eds.). Evolution of Social Behavior in Insects and Arachnids. Cambridge University Press, New York.

Snodgrass, R.E. 1935. Principles of Insect Morphology. McGraw Hill Book Co., Inc., New York. $667 \mathrm{pp}$.

Virkki, N. 1957. Structure of the testis follicle in relation to evolution in the Scarabaeidae (Coleoptera). Can. J. Zool., 35:265-277.

1961. The passalid testis and its structural kinship with testes of other Scarabaeoid beetles. Arch. Soc. Zool. Bot. Fenn. "Vanamo” 16(1): 19-22. 
